\title{
Violencia laboral. Análisis de los factores de riesgo y consecuencias en la vida de las mujeres trabajadoras en la Ciudad de México
}

\author{
Workplace violence. Analysis of risk factors and consequences \\ in the lives of working women in Mexico City
}

\author{
Vianney Berenice Cruz-Arroyo e Irene Casique \\ Instituto Nacional de Desarrollo Social de la Secretaría de Bienestar, México / \\ Centro Regional de Investigaciones Multidisciplinarias de la Universidad \\ Nacional Autónoma de México, Méxicol
}

Resumen

Este artículo analiza la prevalencia de cuatro tipos de violencia contra las mujeres que se presentan en el ámbito laboral, enuncia algunos factores de riesgo asociados con la presencia de tales situaciones violentas y explora la posible conjunción entre las experiencias de violencia laboral y la severidad de algunos padecimientos físicos o psicológicos que presentan mujeres trabajadoras en la Ciudad de México. El artículo está basado en los resultados de una encuesta aplicada a una muestra intencional, no probabilística, de mujeres trabajadoras en empresas e instituciones gubernamentales estatales y federales en la Ciudad de México.

Palabras clave: Violencia laboral contra las mujeres, coerción sexual, acoso de género, padecimientos originados por la violencia laboral, Ciudad de México.

\section{Abstract}

This article analyzes the prevalence of four different kinds of workplace violence against women, enunciates some risk factors associated with the presence of such violent situations and explores the possible conjunction of workplace violence experiences with the severity of some physical or psychological illnesses among female workers in Mexico City. It is based on the results of a survey applied to a purposive, nonprobabilistic, sample of women working at both local or federal government institutions and companies in Mexico City.

Key words: Workplace violence against women, sexual coercion, gender harassment, physical or psychological illnesses among female workers, Mexico City. 


\section{INTRODUCCIÓN}

$\mathbf{L}$

a violencia como acto está presente en la vida cotidiana de miles de personas en el mundo, y nos afecta a todas y todos de manera directa o indirecta y de varias formas. Se manifiesta en las calles, en los hogares, en las escuelas, en los lugares de trabajo y otros centros de concentración de personas, y está tan presente, que a menudo es percibida como un componente ineludible de la condición humana (Organización Panamericana de la Salud, 2002: 3).

En la medida en que se realiza de manera intencional y que tiene como fin someter y controlar a la otra persona, el uso de la violencia está estrechamente relacionado con el ejercicio del poder intrapersonal. La violencia interpersonal se concibe como aquella violencia que ejerce un individuo o grupo de individuos sobre otros con quienes pueden o no tener lazos familiares - dentro o fuera del hogar - como mecanismo para mantener la dominación y el poder frente a alguien que no se reconoce como igual (Ortiz, 2005: 73).

De acuerdo con esta definición de violencia, podemos entender a la violencia de género como aquella violencia que se ejerce desde el modelo hegemónico de masculinidad encarnado mayoritariamente por hombres, pero también por algunas mujeres, contra todas las mujeres y también contra todos los hombres que se adhieren a otros modelos de masculinidad no hegemónica tanto heterosexual como homosexual (Oliver y Rosavalls, 2004: 16).

Pero, si bien la violencia de género puede ser ejercida y recibida por hombres y mujeres, es contra ellas que con mayor frecuencia se comete. En México, la Ley General de Acceso de las Mujeres a una Vida Libre de Violencia, plantea que la violencia contra las mujeres se entiende como "cualquier acción u omisión, basada en su género, que les cause daño o sufrimiento psicológico, físico, patrimonial, económico, sexual o la muerte tanto en el ámbito privado como en el público" (Ley General de Acceso de las Mujeres a una Vida Libre de Violencia, 2007). Asimismo, dicha Ley contempla como ámbitos en los que se presenta la violencia contra las mujeres: el familiar, laboral y docente, comunitario e institucional (Ley General de Acceso de las Mujeres a una Vida Libre de Violencia, 2007).

Considerando estos ámbitos en los que se presenta la violencia de género, la incorporación de las mujeres al mercado laboral extradoméstico - siendo este un espacio simbólicamente de construcción masculina-, ha constituido una manera de subversión a la ideología dominante, lo cual 
puede ser un factor detonante de violencia contra las mujeres, (Díaz, 2005 y Zuñiga, 2005 citados por Molina Armenta, 2010). La mayor presencia de las mujeres en el mercado laboral ha trasladado las situaciones de violencia del espacio privado de los hogares al espacio público de las organizaciones laborales (Lamas, 2004: 191). Y esta violencia laboral, al igual que la que se vive dentro del espacio familiar, representa un problema de salud pública y repercute de manera negativa en el mundo del trabajo.

Si bien no todas las formas de violencia laboral corresponden a violencia de género, diversos estudios en distintas regiones han señalado un mayor riesgo por parte de las mujeres que de los hombres a sufrir violencia laboral (Archer, 1999 y Zapf, Escartin, Einarsen, Hoel y Vartia, 2011, citados por González, 2018).

La urgencia de abordar y prevenir la violencia laboral contra las mujeres, así como proteger sus derechos laborales, quedó plasmada en dos de los objetivos del Desarrollo Sostenible, planteados en la Conferencia de las Naciones Unidas sobre el Desarrollo Sostenible, realizada en Río de Janeiro en 2012: "Eliminar todas las formas de violencia contra todas las mujeres y las niñas en los ámbitos público y privado, incluidas la trata y la explotación sexual y otros tipos de explotación" (Objetivo 5.2) y

Proteger los derechos laborales y promover un entorno de trabajo seguro y sin riesgos para todos los trabajadores, incluidos los trabajadores migrantes, en particular las mujeres migrantes y las personas con empleos precarios (Objetivo 8.8) ( ONU, 2015).

En paralelo, la protección social tanto de hombres como de mujeres en edad de trabajar, incluyendo aspectos como la protección social en caso de accidentes en el trabajo y la creación de puestos de trabajo decente, han sido planteados como requisitos para el logro del Desarrollo Sostenible (OIT, 2017: 83).

En este trabajo nos proponemos analizar diversos aspectos de la violencia laboral hacia las mujeres, a partir de tres objetivos específicos: i) identificar las principales expresiones de violencia laboral contra las mujeres y su prevalencia; ii) identificar algunos factores de riesgo asociados a la violencia laboral en contra de mujeres trabajadoras en la Ciudad de México, y iii) explorar la asociación entre experiencias de violencia laboral y la severidad de padecimientos psicológicos y físicos en las mujeres que trabajan.

Partimos del supuesto de que diversos factores, como las características socioeconómicas de las mujeres, el ambiente laboral, el espacio de trabajo y las relaciones jerárquicas dentro del espacio laboral, se relacionan con 
el riesgo de situaciones de violencia dentro del ámbito laboral; además, la ocurrencia y severidad de algunos padecimientos físicos y psicológicos, se verían incrementados por la experiencia de situaciones de violencia laboral.

Además, a fin de identificar de quién viene o quién ejecuta alguna acción de violencia en contra de las mujeres trabajadoras, consideramos la clasificación de las personas implicadas y el tipo de relación que existe entre ellas que propone la Occupational Safety and Health Administration (OSHA), la cual supone que si bien las manifestaciones de violencia implican un abuso de poder de quien la detenta sobre quien la recibe, la violencia laboral puede ser ejercida tanto por un superior (o jefe) hacia un subordinado, como por compañeros y compañeras (pares) que se encuentran en un mismo nivel jerárquico (Carvajal Orozco y Londoño, 2013). Esta violencia laboral daña la autoestima, la salud, la integridad física y psicológica, la libertad y seguridad de la víctima, e impide su desarrollo profesional, atentando contra la igualdad de oportunidades laborales (Ley General de Acceso de las Mujeres a una Vida Libre de Violencia, 2007).

La Organización Internacional del Trabajo (OIT) define la violencia laboral como toda acción, incidente o comportamiento mediante el cual una persona es agredida, amenazada, humillada o lesionada por otra en el ejercicio de su actividad profesional o como consecuencia directa de la misma que ocurre dentro de un plazo de tiempo razonable (Chapell y Di Martino, 2006: 30).

Normalmente existe la tendencia a asociar la violencia con agresiones físicas, sin embargo, las manifestaciones de violencia en el trabajo suelen ser de distinta naturaleza y pueden adoptar distintas formas, incluidas las no-físicas o psicológicas (Ministerio de la Protección Social, 2004:15). Con frecuencia se trata de la repetición de acciones que pueden verse como menores pero que al acumularse pueden llegar a ser graves, tales como el acoso sexual, la intimidación o acoso laboral (mobbing) (Milczarek, 2010: 18). Así, la violencia en el trabajo puede incluir conductas verbales, físicas o sexuales, amenazantes, intimidatorias, abusivas y acosantes (Ministerio de la Protección Social, 2004: 43).

Entre los incidentes más frecuentes de violencia en el lugar de trabajo se cuentan los abusos verbales y las amenazas. No obstante, en muchas ocasiones o no se les presta atención o se asumen como parte inevitable de la dinámica de trabajo, aunque en realidad pueden tener importantes consecuencias sobre las personas que los sufren e incluso sobre el resto de las y 
los trabajadores que son testigos de la misma (Ministerio de la Protección Social, 2004: 15).

El problema radica en que, a diferencia de las agresiones físicas, los impactos de otros tipos de conductas violentas son difíciles de determinar y a veces los efectos no son inmediatos, además de que están mediados por las distintas percepciones sobre lo que se considera una conducta verbal abusiva o amenazante (como se cita en Pérez y Nogareda, 2004: 15).

Si bien es posible identificar diversos factores que propician o favorecen la violencia contra las mujeres, no es posible señalar una única causa ni un solo efecto final de la misma, por lo que autores como Heise (citada en Olivares e Inchaústegui, 2011: 32) plantean la violencia contra las mujeres como una problemática multicausal.

La complejidad para definir qué son y qué no son las conductas violentas proviene de esta multidimensionalidad del problema; en este trabajo solo consideramos cuatro tipos de violencia que pueden encontrarse en el ámbito laboral, sin negar la existencia de algunas otras formas: i) violencia psicológica o emocional: referida a cualquier acto u omisión que dañe la estabilidad psicológica, puede consistir en negligencia, abandono, humillaciones, marginación, insultos, comparaciones destructivas, entre otras; ii) violencia física: entendida como cualquier acto que inflige daño no accidental, usando la fuerza física o algún tipo de arma u objeto que pueda provocar o no lesiones que sean internas, externas o ambas; iii) violencia económica: es aquella que afecta la supervivencia económica de la víctima, como la percepción de un salario menor por igual trabajo; y iv) violencia sexual: considerando el hostigamiento y acoso sexual como manifestaciones de este tipo de violencia dentro de los espacios laborales. El primero implica una relación de subordinación de la víctima frente al agresor, mientras que, en el acoso sexual, si bien no existe la subordinación, hay un ejercicio abusivo del poder. Ambos se expresan por medio de conductas verbales y/o físicas, relacionadas con la sexualidad y con una connotación lasciva (Ley General de Acceso de las Mujeres a una Vida Libre de Violencia, 2007).

En cuanto a las consecuencias de la violencia que se ejerce en el ámbito laboral, al igual que la violencia que se ejerce en otros ámbitos, puede ocasionar complicaciones directas sobre la salud física, mental y reproductiva de las mujeres (Chapell y Di Martino, 2006: 17-18).

Además, la violencia que se presenta dentro de las organizaciones laborales genera ambientes hostiles, afectando el respeto, la comunicación, el compromiso, el ambiente amigable y la satisfacción de la actividad la- 
boral, que son factores que puntualizan un ambiente laboral favorable, con alta productividad y alto rendimiento, es decir, la violencia laboral va más allá de las relaciones interpersonales entre empleadas y empleados (Alves, 2000: 123-133).

Por otra parte, este tipo de violencia también trae consecuencias económicas, como elevados costos de producción, mayor rotación de personal, disminución o pérdida de la motivación laboral, alteración de las relaciones interpersonales, deterioro del ambiente laboral, aumento de la accidentalidad y ausentismo, entre otros problemas, al interior de las organizaciones (Chapell y Di Martino, 2006: 137).

Dada la magnitud del problema algunos países han comenzado a contabilizar el impacto de la violencia en el mercado laboral y en los sistemas de procuración de justicia y de salud. El Banco Interamericano de Desarrollo (BID), estima que los costos de la violencia contra las mujeres en este ámbito oscilan entre 1.6 y dos por ciento del Producto Interno Bruto (PIB) de los países de la región latinoamericana, por lo que su atención se ha declarado una prioridad en la agenda pública del desarrollo (Instituto Nacional de las Mujeres, 2008: 16).

En México, el problema de la violencia laboral no ha sido estudiado de manera sistemática, y se tienen pocas cifras y referencias sobre el grado, el carácter y las particularidades de este problema. No obstante, existen contados estudios e investigaciones sobre algunas expresiones de violencia laboral, como el acoso laboral (mobbing) y el acoso y hostigamiento sexual, que reflexionan sobre el estado que guarda la legislación nacional en materia de acoso laboral o mobbing y el contraste con el marco jurídico internacional (Centro de Estudios para el Adelanto de las Mujeres y la Equidad de Género, 2011: 10-11). Algunas otras investigaciones, identifican los comportamientos en torno al hostigamiento sexual y otros tipos de violencia como la psicológica, física, verbal y simbólica (Frías, 2011: 332).

Por otro lado, la Encuesta Nacional sobre la Dinámica de las Relaciones en los Hogares 2011, identifica una proporción significativa de la violencia laboral que experimentan las mujeres asociada a la condición de embarazo; los datos indican que a 15.6 por ciento de las mujeres que han trabajado alguna vez o han solicitado empleo les han pedido la prueba de embarazo como requisito de ingreso o permanencia; por otra parte, 3.4 por ciento ha sufrido repercusiones por haberse embarazado, como reducción de su salario, no renovación de su contrato o el despido (INEGI, 2011).

En este sentido, se hacen necesarias las investigaciones en torno a la violencia dentro de los espacios de trabajo que permitan visibilizar y expo- 
ner los factores de riesgo de la violencia que sufren las mujeres dentro de sus espacios de trabajo y además las consecuencias o efectos que esta trae en la vida de las mujeres trabajadoras.

\section{DATOS Y MÉTODOS}

El análisis que realizamos se basa en datos obtenidos a partir de una encuesta intencional, diseñada y aplicada entre mujeres que trabajan en la Ciudad de México, en el año 2012, con el fin de obtener información sobre situaciones de violencia laboral.

La encuesta fue aplicada a mujeres que trabajan en tres dependencias del Gobierno, dos federales y una estatal, así como en cuatro empresas privadas en la Ciudad de México. Tomando en cuenta que el universo de empresas privadas y públicas instaladas en la Ciudad de México es muy grande, se eligieron sólo algunas que nos permitieron facilidad para el acceso y permanencia dentro de las instalaciones para la aplicación del cuestionario, tomando como referencia las características físicas de la organización, número de trabajadoras y trabajadores, ubicación geográfica y tipo de capital.

Las empresas y dependencias elegidas para la aplicación del cuestionario debían cumplir con algunas características específicas, tales como: un número de empleados y empleadas entre 10 y 500 , de los cuáles al menos 40 por ciento fuesen mujeres; que el espacio físico de sus instalaciones permitiera que las mujeres compartieran el espacio con otras u otros empleados hombres y mujeres (por ejemplo las oficinas o las líneas de producción en la fábrica), y finalmente que ofreciesen condiciones adecuadas para el acceso y permanencia dentro de las instalaciones de la organización para la aplicación de la encuesta ${ }^{1}$ (ver Tabla 1 ).

Para la aplicación del cuestionario, se consideró una muestra por cuotas en cada una de las organizaciones laborales, de tal forma que, se encuestó a 199 mujeres trabajadoras entre los 18 y 60 años de edad, que al momento de la encuesta se encontraban laborando en alguna de las empresas seleccionadas, que tuvieran al menos seis meses trabajando en la organización $\mathrm{y}$ en su puesto actual, y por supuesto que aceptaran de manera voluntaria responder el cuestionario.

Previo a la aplicación del cuestionario, se les explicó a estas mujeres que las respuestas y los datos obtenidos serían utilizados con fines meramente académicos, lo anterior para generar confianza entre ellas de que la

\footnotetext{
1 Para la aplicación del cuestionario se solicitó por escrito el permiso para la entrada y permanencia dentro de las instalaciones de las organizaciones con el fin de recabar los datos contenidos en el cuestionario.
} 
información sería confidencial y lograr una buena tasa de respuesta. De esta manera se logró que al menos 50 por ciento de las trabajadoras en cada una de las de las instituciones seleccionadas aceptaran participar respondiendo al cuestionario (ver Tabla 1).

Por lo anterior, se trata de una muestra intencional, no probabilística, que no nos permite hacer generalizaciones para poblaciones más amplias, más allá de las mujeres incluidas en la muestra, pero nos proporciona información exploratoria sobre el problema.

La encuesta explora cuatro tipos de violencia contra las mujeres dentro del ámbito laboral: violencia física, violencia emocional o psicológica, violencia sexual (acoso y hostigamiento sexual) y violencia económica. Además de conocer la prevalencia de estos tipos de violencia laboral, la encuesta permite identificar quiénes son los perpetradores de la misma (jefe, jefa, compañero/os, compañeras/as u otro/s), y explorar algunos de los factores de riesgo para cada uno de estos tipos de violencia laboral, así como algunas consecuencias psicológicas y físicas derivadas de situaciones de violencia.

El cuestionario está formado por ocho secciones que proporcionan información sobre las características personales de las mujeres; el contexto laboral; el ambiente laboral; el espacio laboral; las situaciones de violencia emocional o psicológica y violencia económica; identificación de situaciones de violencia sexual y física; mecanismos de queja y denuncia de situaciones de violencia laboral y, las consecuencias psicológicas y físicas derivadas de alguna situación de violencia dentro del espacio laboral. Asimismo, incluye preguntas cerradas sobre el ambiente laboral, espacio laboral y las situaciones de violencia laboral que han padecido las mujeres encuestadas, con alternativas de respuestas que plantean una escala de posiciones (de acuerdo o desacuerdo, de frecuencia de ocurrencia de una determinada situación, de temporalidad, de tipo de agresor, entre otros) en las que las mujeres encuestadas ubicaron la alternativa que más se acercaba a su situación.

\section{Indicadores de violencia laboral (variables dependientes)}

Para la construcción de las variables analizadas, partimos de la definición de violencia laboral, considerada como aquella que se manifiesta a través de acciones realizadas por una persona en contra de otra dentro del espacio de trabajo y que dañan su dignidad e integridad física, sexual, psicológica, económica o social (Chapell y Di Martino, 2006: 30). 
Violencia laboral. Análisis de los factores de riesgo y consecuencias en la vida de las mujeres ... / V.B. CRUZ ARROYO e I. CASIQUE

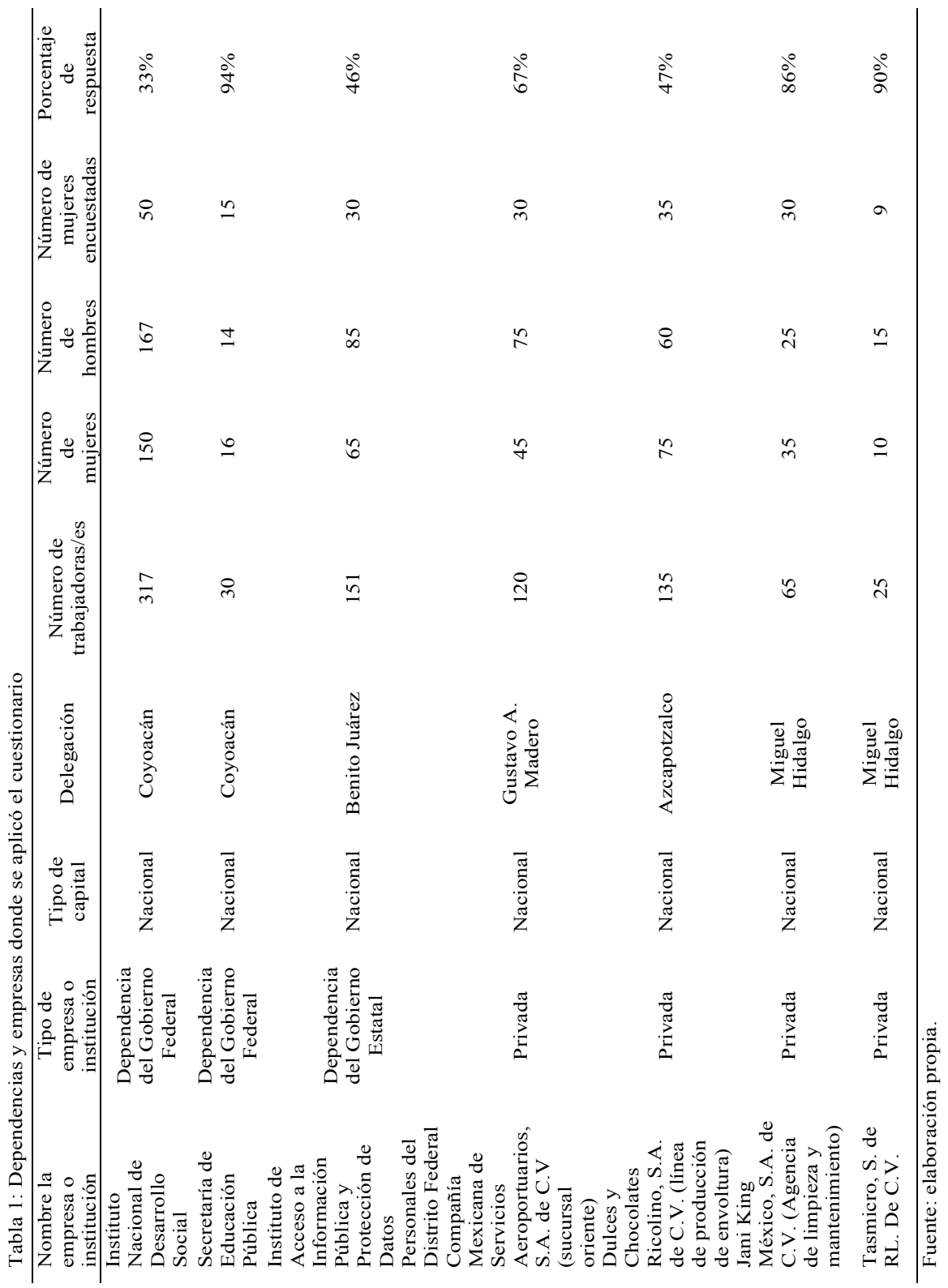


En este sentido, se consideraron como situaciones de violencia laboral cualquiera de las siguientes acciones:

a. Violencia física: empujones, golpes, patadas, bofetadas, entre otros.

b. Violencia emocional, psicológica y mobbing: intimidación, humillaciones verbales, comentarios sexistas, amenaza de violencia física, críticas negativas al desempeño laboral, a la vestimenta, a la apariencia física.

c. Violencia sexual: acoso, hostigamiento sexual, miradas lascivas, tocamientos, forzar física o emocionalmente a la mujer a la relación sexual, solicitar favores sexuales a cambio de mejores condiciones laborales.

d. Violencia económica: reducción del salario y/o prestaciones, dificultar u obstaculizar promociones o ascensos laborales.

Diversas preguntas del cuestionario buscan identificar si las mujeres habían sufrido algún tipo de violencia física, psicológica o emocional (incluyendo el mobbing), violencia sexual y violencia económica; cuyas respuestas se utilizaron para la construcción de indicadores dicotómicos que se incorporaron como variables dependientes al análisis de regresión.

Así para la violencia física, se preguntó a las mujeres sí habían sido agredidas físicamente en su trabajo, generando con esta información un indicador dicotómico que adopta el valor de 1 si la mujer indicó una vez, varias veces o siempre, y el valor de 0 para aquellas cuya respuesta fue nunca.

Para la violencia emocional, psicológica y mobbing, se generó una variable dicotómica, a la cual se asignó un valor de 1 cuando la respuesta fue una vez, varias veces o siempre a alguna de las preguntas planteadas (ver Tabla 2) y un valor de cero cuando la respuesta fue nunca.

La variable violencia sexual, se conforma de varios items que permitieron identificar las situaciones de acoso y hostigamiento sexual que han vivido las mujeres encuestadas.

Dado que no existe una única definición de hostigamiento y acoso sexual, para este trabajo tomamos la conceptualización de Gruber (citado en Frías, 2011), que combina la naturaleza de los actos con la intención detrás de los mismos y los clasifica en peticiones verbales, comentarios verbales y manifestaciones no verbales.

Es así que, la primera dimensión de violencia sexual que distinguimos es el Acoso basado en el género, se refiere a aquellos comportamientos groseros u ordinarios de carácter verbal, físico y simbólico que transmiten actitudes hostiles, ofensivas y misóginas, cuyo fin es excluir a las mujeres 
de un determinado ámbito de trabajo; la segunda dimensión de violencia sexual es la Atención sexual no buscada, que consiste en atención sexual no recíproca y no deseada por quien es objeto de la misma; y finalmente distinguimos la Coerción sexual, como aquellas proposiciones sutiles o explícitas de obtener mejores condiciones de empleo a un intercambio de naturaleza sexual (Frías, 2013: 80).

Tabla 2: Preguntas para captar violencia psicológica laboral

1. ¿No le han dado instrucciones precisas para realizar sus labores?

2. ¿Le han criticado de manera negativa y sin razón su trabajo?

3. ¿La han limitado a realizar tareas inferiores a sus capacidades?

4. ¿Le han impedido participar de la toma de decisiones relacionadas directamente con sus tareas y en las que Ud. debería participar?

5. ¿Le han reducido responsabilidades y tareas sin justificación ni previo aviso a fin de no permitir su desarrollo dentro de la empresa o institución?

6. ¿Le han incrementado responsabilidades y tareas sin justificación?

7. ¿Trabaja o ha tenido que trabajar horas extras sin su consentimiento?

8. ¿Le han obstaculizado o impedido alguna promoción o ascenso por ser mujer?

9. ¿Ha sido descalificada - de manera injusta-en sus tareas o responsabilidades laborales?

10. ¿Constantemente controlan, supervisan o monitorean su trabajo de manera negativa?

11. ¿Le han escondido información, documentos, artículos o herramientas de trabajo?

12. ¿La llaman con algún tipo de apodo que le molesta?

13. ¿Le han hecho comentarios negativos sobre su vestimenta?

14. ¿Le han criticado de manera negativa y sin razón su trabajo?

15. ¿Le han prohibido hablar con sus compañeras/os?

16. ¿La han aislado de sus compañeras/os?

17. ¿En su trabajo la han humillado o denigrado? 
Una vez definidas estas tres dimensiones, se generaron tres indicadores conformados por variables dicotómicas, tomando los ítems utilizados para identificar la violencia sexual, pero haciendo una selección de acuerdo con las definiciones de cada dimensión dando un valor de 1 para aquellas mujeres que respondieron haber sufrido una vez, varias veces o siempre a cualquiera de las situaciones de este tipo, y un valor de 0 para aquellas que contestaron que nunca. El primer indicador Acoso de género, se conforma por los items de la Tabla 3:

Tabla 3: Preguntas para detectar Acoso de género

1. ¿Le han hecho comentarios sexistas?

2. ¿Le han contado chistes o hecho bromas sexuales que le resultaron ofensivas?

3. ¿Le han hecho comentarios ofensivos o vulgares sobre su apariencia, cuerpo o sexo?

Fuente: encuesta propia.

Para el indicador de Atención sexual no buscada, se usaron los ítems de la Tabla 4:

La última de las dimensiones que conforman la variable violencia sexual, se refiere a la Coerción Sexual, cuyo indicador se construyó a partir de las variables dicotómicas de la Tabla 5:

El indicador de violencia económica se basa en la pregunta planteada a las mujeres respecto a sí les habian reducido su salario o prestaciones sin previo aviso y sin justificación. Esta variable es una variable dicotómica que adopta el valor de 1 si la mujer indicó una vez, varias veces o siempre le han reducido su salario y prestaciones; y 0 para aquellas trabajadoras que contestaron que nunca.

\section{Variables independientes}

En primer lugar, se incluyen en el análisis como variables independientes algunos indicadores relacionados con diversas características socioeconómicas de las mujeres de la muestra tales como edad, estado civil, escolaridad y número de hijas e hijos. Asimismo, incorporamos indicadores sobre algunas condiciones laborales de las mujeres como: antigüedad en el trabajo, tipo de contrato, jornada de trabajo y número de días a la semana que trabajan. Finalmente, incluimos algunas variables referidas a carac- 
Violencia laboral. Análisis de los factores de riesgo y consecuencias en la vida de las mujeres ... / V.B. CRUZ ARROYO e I. CASIQUE

terísticas de los lugares de trabajo, tales como: número de personas que laboran, sexo mayoritario en el lugar de trabajo y sexo del jefe jerárquico.

Tabla 4: Preguntas para detectar Atención Sexual no buscada

1. ¿La han mirado de manera obscena o de alguna forma que la haya hecho sentir incómoda?

2. ¿Le han mostrado o usado delante de usted imágenes u objetos con carácter sexual (¿fotos, videos, juguetes, etc.?

3. ¿La han tocado (caricias, nalgadas, palmadas, pellizcos), abrazado, metido mano o besado sin su consentimiento?

4. ¿La han acosado mediante correos electrónicos, por teléfono o alguna otra forma?

5. ¿Le han enviado regalos con alguna connotación sexual (ropa interior, juguetes, películas, revistas, etc.) que la hayan hecho sentir incómoda?

6. ¿La han acosado para tener alguna cita fuera de su lugar de trabajo?

Fuente: encuesta propia.

Tabla 5: Preguntas para detectar Coerción Sexual

1. ¿La han forzado a tener relaciones sexuales?

2. ¿Le han propuesto mejores condiciones o ascensos laborales a cambio de tener relaciones sexuales?

3. ¿La han forzado $\mathrm{u}$ obligado a tener relaciones sexuales a cambio de mejores condiciones o ascensos laborales?

4. ¿La han obligado a tener relaciones sexuales a cambio de mantener su trabajo?

5. ¿Han tomado represalias o la han castigado por haberse negado a tener algún acercamiento sexual o mantener tener relaciones sexuales?

Fuente: encuesta propia.

Adicionalmente, se generaron como variables independientes, para el análisis de las consecuencias de la violencia laboral, dos índices a fin de explorar la severidad de los padecimientos emocionales y físicos que su- 
fren las mujeres derivados de alguna situación de violencia laboral. Posteriormente, estos dos índices son empleados como variables dependientes en dos modelos de regresión lineal que desarrollamos para explorar la severidad de las consecuencias psicológicas y físicas de la violencia laboral.

Para la construcción del índice de severidad de padecimientos psicológicos y emocionales se generaron variables categóricas derivadas de la respuesta a la pregunta: En el último año y derivado del ambiente, espacio laboral, relaciones con su/s superior/es, compañeras/os o de tensiones y problemas en el trabajo ¿Ud. ha sufrido o padecido alguno/os de los siguientes problemas?: ansiedad, miedo, estrés, problemas de sueño (insomnio), depresión, irritabilidad, dificultad de concentración, ideas suicidas y apatía o distanciamiento de los demás. Para cada ítem, se dio un valor de 1 cuando la respuesta fue una vez, 2 para varias veces y 3 cuando ocurría siempre, mientras que para aquellas que contestaron nunca, se asignó el valor 0 . Una vez generadas las variables, se evaluó la consistencia interna de las mismas, las cuales presentan un Alpha de Cronbach de 0.86. Además, estos factores fueron ponderados y estandarizados obteniendo un rango de valores que van de 0 a 1 , donde los valores cercanos a 0 indican una baja severidad en algunos de los padecimientos psicológicos y emocionales derivados de situaciones de violencia que sufrieron las mujeres en su lugar de trabajo, mientras que el valor de 1 representa un grado mayor en la severidad de estos padecimientos.

De manera similar el índice de severidad de padecimientos físicos se generó a partir de variables categóricas basadas en las respuestas a la pregunta: En el último año y derivado del ambiente, espacio laboral, relaciones con su/s superior/es, compañeras/os o de tensiones y problemas en el trabajo ¿Ud. ha sufrido o padecido alguno/os de los siguientes problemas?: dolores musculares, pérdida de audición, problemas de la vista, dolores de cabeza y migraña, trastornos gastrointestinales, cansancio permanente y consumo excesivo de alcohol o drogas. Para cada item, se dio un valor de 1 cuando la respuesta fue una vez, 2 para varias veces y 3 cuando ocurría siempre, mientras que para aquellas que contestaron nun$c a$, se asignó el valor 0 . Generadas las variables, se evaluó su consistencia mediante Alpha de Cronbach (0.80); posteriormente mediante análisis factorial se obtuvieron dos factores que fueron ponderados y estandarizados, y cuyo rango va de 0 a 1 , donde los valores cercanos a 0 indican un grado menor de severidad en algunos de los padecimientos físicos derivados de situaciones de violencia que sufrieron las mujeres en su lugar de trabajo, 
mientras que el valor de 1 representa un grado mayor en la severidad de estos padecimientos.

Se generó además, un índice referente a la satisfacción con el ambiente laboral, a partir de nueve preguntas como: ¿Le gusta su empleo?, ¿Le gusta el ambiente laboral que hay en su empresa o institución?, ¿se siente a gusto con su puesto de trabajo?, ¿El trato entre jefes/as y subordinados/ as es amable y respetuoso?; las cuales dan cuenta de la percepción que las mujeres tienen acerca del trato y las relaciones entre las personas con las que laboran, así como respecto a las actividades que realizan en sus lugares de trabajo. El Alpha de Cronbach correspondiente para este índice es de 0.76 , indicando una buena consistencia entre los items.

De igual forma, se estimó e incluyó un índice de satisfacción con el espacio laboral, que muestra la percepción que tienen las mujeres acerca de las instalaciones de la empresa, las medidas de seguridad, las condiciones del espacio de trabajo, el mobiliario, equipo y herramientas de trabajo. Este índice se generó a partir de preguntas como: ¿Las instalaciones de la empresa o institución están en buenas condiciones?, ¿Usted cuenta con el espacio adecuado para desarrollar sus actividades laborales?, ¿Usted cuenta con el mobiliario, equipo y herramientas adecuadas para desarrollar sus actividades laborales? Este índice arroja un Alpha de Cronbach de 0.76 .

Los valores de estos dos índices también fueron estandarizados entre 0 y 1 , donde los valores cercanos a 1 indican mayor satisfacción con el ambiente y el espacio laboral, mientras que los cercanos a cero indican una menor satisfacción.

En la Tabla 6 se presenta la distribución de frecuencias (o media) de las variables utilizadas en el análisis de la muestra de mujeres empleadas, distinguiendo entre mujeres que han experimentado violencia laboral $\mathrm{y}$ aquellas que no. Sólo tres variables evidencian diferencias significativas entre las mujeres que no han experimentado violencia laboral y las que sí han recibido violencia laboral. En primer lugar, se observa que las mujeres que han vivido violencia laboral presentan una mayor concentración en las oficinas como lugar de trabajo, en comparación con aquellas que trabajan en otros espacios ( 93.18 por ciento vs 79.35 por ciento). Por otra parte, entre las mujeres que han sufrido violencia laboral es mayor el porcentaje en aquellas que tienen un contrato temporal que en aquellas que no (26.4 vs 13.64 por ciento). 
Tabla 6: Características generales de la muestra (Distribución o media de las variables)

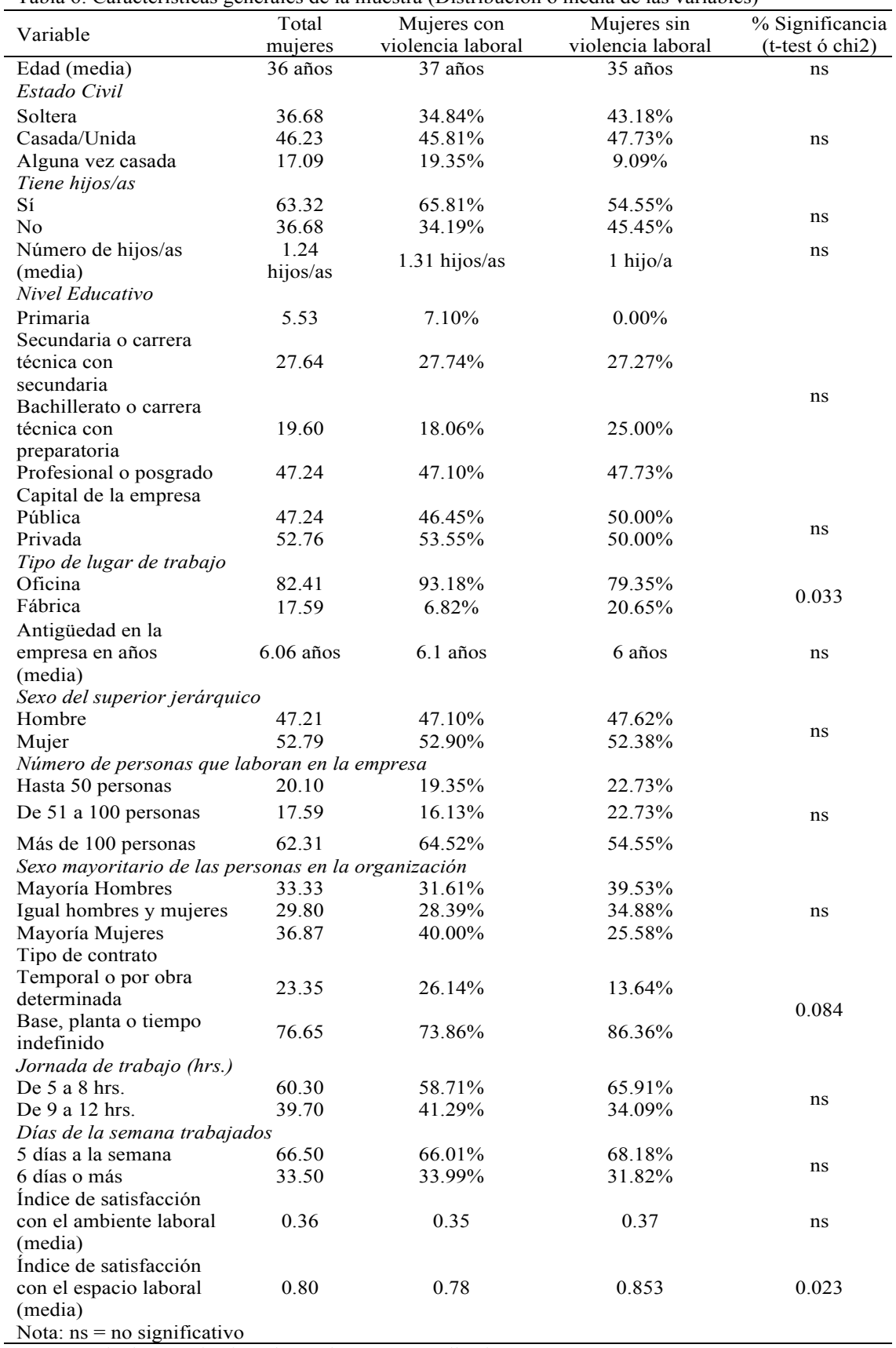

Fuente: cálculos propios basados en la encuesta aplicada. 
Finalmente, el índice de satisfacción con el espacio laboral es significativamente más alto entre las mujeres libres de violencia laboral que en aquellas que si la han padecido ( 0.85 vs 0.78 , respectivamente), indicando que entre las primeras hay una mayor satisfacción con las condiciones del espacio en que laboran (ver Tabla 6).

\section{Prevalencia de la Violencia laboral entre mujeres trabajadoras en la Ciudad de México}

Los datos de la encuesta permiten obtener la prevalencia de los distintos tipos de violencia laboral experimentados por las mujeres de esta muestra a lo largo de sus vidas laborales y durante el último año (ver Tabla 7). La violencia emocional y el acoso de género son las dos expresiones de violencia laboral más frecuentemente experimentadas por las mujeres de esta muestra. La atención sexual no buscada se registra en menor medida, sin embrago, la misma ha afectado a más de 40 por ciento de estas mujeres en algún momento. Los tipos de violencia laboral que presentan más baja frecuencia son la violencia física y la económica.

Tabla 7: Prevalencia de los distintos tipos de violencia laboral

\begin{tabular}{lcr}
\hline Tipo de violencia & Alguna vez & Último año \\
\hline Violencia Emocional o Psicológica & $62.81 \%$ & $55.78 \%$ \\
Violencia Económica & $4.52 \%$ & $3.02 \%$ \\
Violencia Física & $4.52 \%$ & $3.02 \%$ \\
Acoso de género & $57.79 \%$ & $49.25 \%$ \\
Atención Sexual no buscada & $40.70 \%$ & $29.15 \%$ \\
Coerción Sexual & $7.04 \%$ & $4.52 \%$ \\
\hline
\end{tabular}

Fuente: cálculos propios basados en la encuesta aplicada.

En cuanto a quiénes son las personas agresoras, en la Tabla 8 se muestra que la violencia emocional, la violencia física y la coerción sexual son mayormente ejercidas por superiores jerárquicos $(57.5,75$ y 66.67 por ciento, respectivamente), en tanto que el acoso sexual y la atención sexual no buscada son ejercidas con mayor frecuencia por compañeros o compañeras de trabajo (77.19 y 55.56 por ciento) respectivamente. 


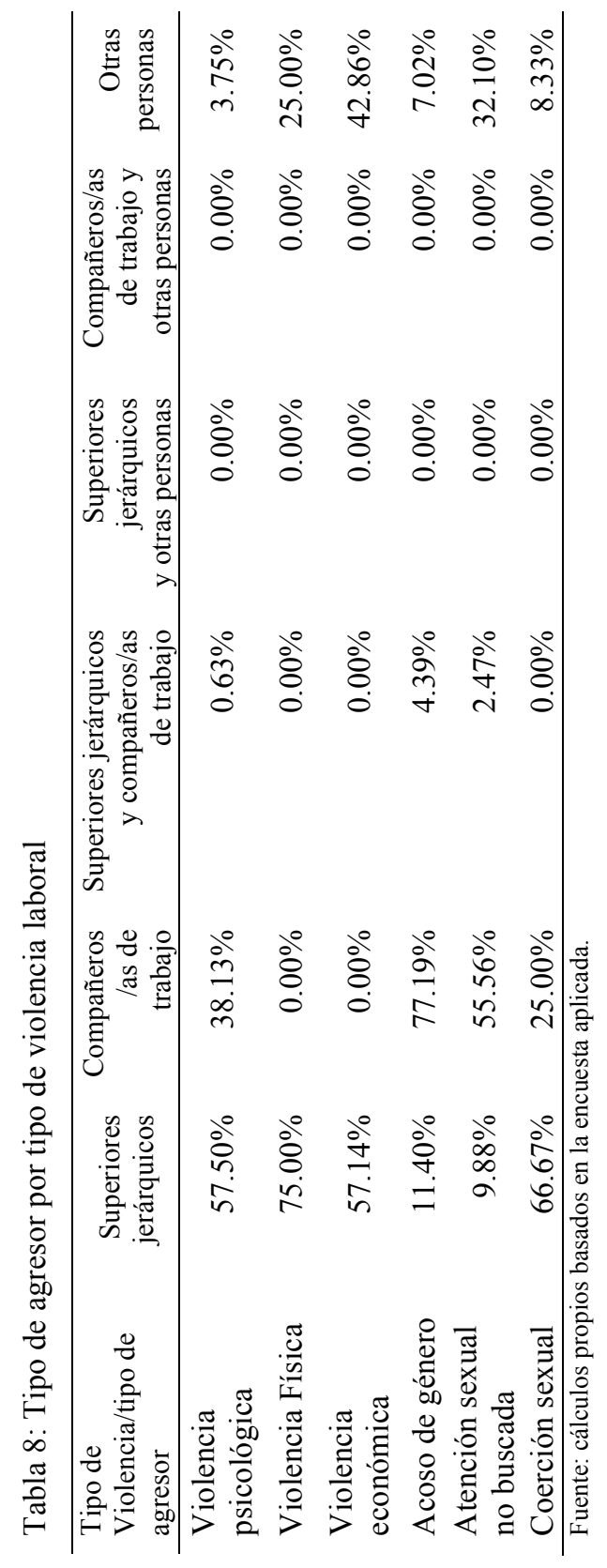


Violencia laboral. Análisis de los factores de riesgo y consecuencias en la vida de las mujeres ... / V.B. CRUZ ARROYO e I. CASIQUE

Análisis multivariado de los factores asociados AL Riesgo de SUFRIR VIOLENCIA LABORAL EN MUJERES TRABAJAdORAS EN LA CIUDAD DE MÉXICO

Para examinar los factores asociados al riesgo de violencia laboral estimamos tres modelos de regresión logit multivariados, uno para cada una de las tres expresiones más frecuentes de violencia laboral identificadas: la violencia laboral psicológica, el acoso de género y la atención sexual no buscada.

En el primer modelo empleamos como variable dependiente la variable violencia laboral emocional. Los resultados se presentan en el Modelo 1 de la Tabla 9, y podemos observar que, del conjunto de variables incluidas en el modelo, solo cuatro se asocian significativamente al riesgo de violencia laboral emocional en contra de las mujeres.

El estado civil de las mujeres presenta una asociación marginalmente significativa $(\mathrm{p} \leq 0.10)$ observándose que el riesgo de violencia laboral psicológica para las mujeres casadas o unidas es 55 por ciento menor que la correspondiente para las mujeres solteras. Por otra parte, el lugar de trabajo también resulta marginalmente significativo, observándose que las mujeres que trabajan en una fábrica corren 8.8 veces más riesgo de sufrir este tipo de violencia que las mujeres que trabajan en una oficina.

Los índices de satisfacción con el ambiente laboral y de satisfacción con el espacio de trabajo muestran también una relación negativa y significativa con el riesgo de violencia psicológica laboral, de manera que por cada incremento unitario en estos índices la probabilidad de violencia psicológica disminuye en 90 y 95 por ciento, respectivamente (ver Tabla 8).

Los resultados de la regresión multivariada para el acoso de género se muestran en el Modelo 2 (Tabla 9). Podemos observar que el lugar de trabajo se asocia de manera significativa con el riesgo de experimentar este tipo de violencia, y que aquellas mujeres cuyo lugar de trabajo es una fábrica tienen mayor riesgo de sufrir acoso de género (19 veces más) que el riesgo que presentan las mujeres que laboran en una oficina.

El número de personas que laboran en la empresa también aparece como un factor de riesgo del acoso de género en los espacios de trabajo: las mujeres que laboran en empresas con más de 100 personas tienen un riesgo menor (razón de probabilidad 68 por ciento menor) que aquellas mujeres que laboran en empresas pequeñas con un máximo de 50 empleados. 
Tabla 9: Resultados del Modelo de regresión logística multivariada por tipo de violencia laboral

\begin{tabular}{|c|c|c|c|c|c|c|}
\hline \multirow[t]{2}{*}{ Variable } & \multicolumn{2}{|c|}{$\begin{array}{l}\text { Modelo 1 } \\
\text { Violencia } \\
\text { Psicológica }\end{array}$} & \multicolumn{2}{|c|}{$\begin{array}{c}\text { Modelo } 2 \\
\text { Acoso de género }\end{array}$} & \multicolumn{2}{|c|}{$\begin{array}{c}\text { Modelo } 3 \\
\text { Atención sexual no } \\
\text { buscada }\end{array}$} \\
\hline & O.R. & $\begin{array}{c}\mathrm{P} \\
\text { (sign.) }\end{array}$ & O.R. & $\begin{array}{c}\mathrm{P} \\
\text { (sign.) }\end{array}$ & O.R. & $\begin{array}{c}\mathrm{P} \\
\text { (sign.) }\end{array}$ \\
\hline Edad & 1.023 & ns & 1.041 & ns & 1.011 & $\mathrm{~ns}$ \\
\hline \multicolumn{7}{|c|}{ Nivel educativo alcanzado } \\
\hline Primaria (ref.) & 1 & & 1 & & 1 & \\
\hline Secundaria & 0.387 & ns & 1.688 & ns & 1.329 & ns \\
\hline Bachillerato & 0.5 & ns & 1.544 & ns & 0.796 & ns \\
\hline $\begin{array}{l}\text { Profesional o } \\
\text { posgrado }\end{array}$ & 0.894 & ns & 1.186 & ns & 1.544 & ns \\
\hline \multicolumn{6}{|l|}{ Estado civil } & \\
\hline Casada/Unida & 0.447 & 0.098 & 0.449 & 0.097 & 1.103 & ns \\
\hline \multicolumn{6}{|l|}{ Tiene hijas/os } & ns \\
\hline No (ref.) & 1 & & 1 & & 1 & \\
\hline $\begin{array}{l}\text { Sí } \\
\text { Lugar de trabajo }\end{array}$ & \multicolumn{5}{|c|}{ Lugar de trabajo } & 0.005 \\
\hline Oficina (ref.) & 1 & & 1 & & 1 & \\
\hline Fábrica & 8.826 & 0.052 & 18.676 & 0.011 & 3.476 & 0.048 \\
\hline \multicolumn{7}{|l|}{ Capital de la empresa } \\
\hline Pública (ref.) & 1 & & 1 & & 1 & \\
\hline Privada & 1.873 & ns & 1.357 & ns & 1.314 & ns \\
\hline $\begin{array}{l}\text { Antigüedad en la } \\
\text { empresa }\end{array}$ & 1.047 & ns & 0.926 & 0.039 & 0.96 & $\mathrm{~ns}$ \\
\hline \multicolumn{7}{|c|}{ Número de personas que laboran en la empresa } \\
\hline $\begin{array}{l}\text { Hasta } 50 \text { personas } \\
\text { (ref.) }\end{array}$ & 1 & & 1 & & 1 & \\
\hline $\begin{array}{l}\text { De } 51 \text { a } 100 \\
\text { personas }\end{array}$ & 0.75 & ns & 0.292 & $\mathrm{~ns}$ & 0.915 & ns \\
\hline $\begin{array}{l}\text { Más de } 100 \\
\text { personas }\end{array}$ & 0.659 & ns & 0.321 & 0.035 & 0.325 & 0.052 \\
\hline \multicolumn{7}{|l|}{ Sexo del jefe } \\
\hline Hombre (ref.) & 1 & & 1 & & 1 & \\
\hline Mujer & 0.845 & ns & 0.902 & ns & 1.08 & ns \\
\hline \multicolumn{7}{|c|}{ Sexo mayoritario en la empresa } \\
\hline $\begin{array}{l}\text { Mayoría hombres } \\
\text { (ref.) }\end{array}$ & 1 & & 1 & & 1 & \\
\hline $\begin{array}{l}\text { Igual hombres y } \\
\text { mujeres }\end{array}$ & 0.931 & ns & 1.073 & ns & 0.215 & 0.017 \\
\hline Mayoría mujeres & 0.753 & ns & 0.585 & ns & 0.714 & ns \\
\hline \multicolumn{7}{|c|}{ Días de la semana trabajados } \\
\hline 5 días (ref.) & 1 & & 1 & & 1 & \\
\hline 6 o más días & 0.52 & ns & 0.249 & 0.096 & 0.499 & ns \\
\hline \multicolumn{7}{|c|}{ Jornada de trabajo (hrs.) } \\
\hline De 5 a 8 horas (ref.) & 1 & & 1 & & 1 & \\
\hline \multicolumn{6}{|l|}{ Tipo de contrato } & 0.081 \\
\hline $\begin{array}{l}\text { Temporal o por obra } \\
\text { determinada (ref.) }\end{array}$ & 1 & & 1 & & 1 & \\
\hline $\begin{array}{l}\text { De base, planta o } \\
\text { tiempo indefinido } \\
\text { Índice de }\end{array}$ & 1.029 & ns & 1.961 & ns & 0.813 & $\mathrm{~ns}$ \\
\hline $\begin{array}{l}\text { satisfacción con el } \\
\text { espacio laboral } \\
\text { Índice de }\end{array}$ & 0.101 & 0.029 & 0.08 & 0.018 & 0.532 & $\mathrm{~ns}$ \\
\hline $\begin{array}{l}\text { satisfacción con el } \\
\text { ambiente laboral }\end{array}$ & 0.052 & 0.008 & 0.121 & 0.05 & 0.211 & $\mathrm{~ns}$ \\
\hline $\mathrm{N}$ & & 191 & & 191 & & 191 \\
\hline Log Likelihood & & 115.363 & & -114.514 & & 95.056 \\
\hline Pseudo $\mathrm{R}^{2}$ & & 0.124 & & 0.135 & & 0.177 \\
\hline
\end{tabular}


Adicionalmente, la antigüedad en la empresa muestra una asociación negativa con el riesgo de acoso de género, disminuyendo la razón de probabilidad en siete por ciento por cada año adicional de trabajo de la mujer en la empresa.

Por otra parte, a mayor satisfacción con el ambiente laboral menor riesgo de experimentar acoso de género: por cada incremento unitario en el índice de satisfacción con el ambiente laboral se reduciría la razón de probabilidad de sufrir acoso de género de las mujeres en 92 por ciento.

Algunas otras variables incluidas en el análisis muestran asociaciones marginalmente significativas con el riesgo de acoso de género como: el estado civil, se observa que aquellas mujeres actualmente casadas o unidas y aquellas alguna vez unidas presentan menores riesgos de sufrir acoso de género que las mujeres solteras ( 55 y 65 por ciento menos, respectivamente).

Por otra parte, los resultados del modelo correspondiente a la atención sexual no buscada (Modelo 3, Tabla 9), indican que solamente tres de las variables incluidas presentan asociaciones significativas con el riesgo de este tipo de violencia laboral. En primer lugar, las mujeres con hijas e hijos tienen un riesgo 80 por ciento menor de sufrir esta forma de violencia que a aquellas mujeres sin hijas e hijos. Las fábricas como lugar de trabajo conllevan un mayor riesgo para las mujeres de sufrir atención sexual, 3.5 veces mayor al correspondiente para las mujeres que trabajan en oficinas. Y el trabajo en empresas con una proporción similar de hombres y de mujeres empleados supone una reducción del riesgo de atención sexual no buscada hacia las mujeres que allí trabajan, 79 por ciento menor, que el riesgo correspondiente a aquellas mujeres que trabajan en lugares en que la mayoría de empleados son hombres.

Otras dos variables evidencian asociaciones marginalmente significativas con el riesgo de recibir atención sexual no buscada. En primer lugar, el número de empleadas y empleados, mostrando que en aquellos lugares donde laboran más de 100 personas la probabilidad de atención sexual no buscada disminuye en 67 por ciento en comparación con los lugares de trabajo que tienen 50 o menos empleados. Por otra parte, una jornada laboral larga (alrededor de 9 horas o más al día) plantea una razón de probabilidad de atención sexual no buscada 2.5 veces mayor comparada con la probabilidad correspondiente a aquellas mujeres que tienen una jornada laboral más corta, de cinco a ocho horas diarias (ver Tabla 9, modelo 3). 
SEVERIDAd de LOS PADECIMIENTOS FÍSICOS Y PSICOLÓGICOS ASOCIADOS A LA Violencia laboral en MUJeres trabajadoras en la CiUdAd de MÉXICO

Diversos padecimientos físicos y psicológicos son experimentados por las mujeres de la muestra como consecuencia de algunas condiciones y tensiones laborales. Es importante notar que estos padecimientos pueden ocurrir como consecuencia de otras situaciones, además de la violencia laboral, y por lo tanto pueden presentarse tanto entre mujeres que han padecido violencia laboral como en aquellas que no. Sin embargo, se corrobora en las mujeres de esta muestra que la prevalencia de los padecimientos es sistemática y significativamente mayor entre aquellas que viven o han vivido alguna situación de violencia, en comparación con aquellas que manifestaron lo contario (ver Tabla 10).

La severidad de los padecimientos físicos y psicológicos que las mujeres encuestadas manifestaron sufrir o haber sufrido derivados de alguna situación de violencia laboral fueron analizados mediante dos modelos de regresión lineal, a fin de identificar algunos factores de riesgo en la ocurrencia de éstos.

En la Tabla 11 podemos observar los resultados obtenidos en los dos modelos estimados. El primer modelo presenta los resultados de la estimación de los factores asociados a la severidad de los padecimientos psicológicos derivados de situaciones de violencia en el trabajo. Este modelo explica 20 por ciento de la varianza del conjunto de variables que aparecen en este modelo, sólo tres muestran asociaciones significativas con la severidad de los padecimientos psicológicos que experimentan las mujeres.

En primer lugar, el estado civil de las mujeres presenta una asociación marginalmente significativa con el índice, ya que para aquellas mujeres que están casadas o unidas la severidad de los padecimientos psicológicos disminuye un 0.07 , en comparación con las mujeres solteras.

Por otro lado, la jornada de trabajo de 9 a 12 horas incrementa la severidad de padecimientos psicológicos en 0.12 en contraste con aquellas mujeres que laboran de cinco a ocho horas diarias; una jornada larga de trabajo expone en mayor medida a las mujeres a más o más graves consecuencias, en este caso psicológicas, de la violencia laboral (ver Tabla 11).

Además, la variable referente a la presencia de violencia laboral, también se relaciona de manera significativa con la severidad de los padecimientos psicológicos, ya que para aquellas mujeres que han sufrido alguna situación de violencia laboral se incrementa el valor de este índice en 0.13. 
En cuanto a los padecimientos físicos de la violencia laboral, el modelo estimado explica 18 por ciento de la varianza de la severidad de los padecimientos físicos. Del conjunto de variables que aparecen en el modelo, cuatro de ellas presentan asociación significativa con esta variable.

Tabla 10: Distribuciones de frecuencia de los padecimientos psicológicos y físicos asociados a la violencia laboral

\begin{tabular}{|c|c|c|c|c|}
\hline Efecto & $\begin{array}{l}\text { Total de } \\
\text { mujeres de } \\
\text { la muestra }\end{array}$ & $\begin{array}{c}\text { Mujeres sin } \\
\text { violencia } \\
\text { laboral }\end{array}$ & $\begin{array}{l}\text { Mujeres con } \\
\text { violencia } \\
\text { laboral }\end{array}$ & $\begin{array}{c}\mathrm{Chi}^{2} \\
\text { (signf.) }\end{array}$ \\
\hline \multicolumn{5}{|l|}{ Padecimientos psicológicos } \\
\hline Ansiedad & 21.11 & 4.55 & 25.81 & $* *$ \\
\hline Miedo & 14.57 & 2.27 & 18.06 & $* *$ \\
\hline Estrés & 77.39 & 54.55 & 82.87 & $* * *$ \\
\hline $\begin{array}{l}\text { Problemas de sueño } \\
\text { (insomnio) }\end{array}$ & 21.61 & 2.27 & 27.1 & $* * *$ \\
\hline Depresión & 21.11 & 2.27 & 26.45 & $* *$ \\
\hline Irritabilidad & 21.61 & 6.82 & 25.81 & $* *$ \\
\hline $\begin{array}{l}\text { Dificultad de } \\
\text { concentración }\end{array}$ & 39.2 & 22.73 & 43.87 & $*$ \\
\hline Ideas suicidas & 4.02 & 2.27 & 4.52 & ns \\
\hline $\begin{array}{l}\text { Apatía o distanciamiento } \\
\text { de los demás }\end{array}$ & 15.58 & 4.55 & 18.71 & $*$ \\
\hline \multicolumn{5}{|l|}{ Padecimientos físicos } \\
\hline $\begin{array}{l}\text { Dolores musculares } \\
\text { (espalda, cuello, } \\
\text { articulaciones) }\end{array}$ & 40.2 & 22.73 & 45.16 & $* *$ \\
\hline Pérdida de audición & 5.03 & 0 & 6.45 & ns \\
\hline Problemas de vista & 18.09 & 6.82 & 21.29 & $*$ \\
\hline $\begin{array}{l}\text { Dolores de cabeza y } \\
\text { migraña }\end{array}$ & 39.2 & 27.27 & 42.58 & ns \\
\hline $\begin{array}{l}\text { Trastornos } \\
\text { gastrointestinales (colitis, } \\
\text { gastritis, estreñimiento, } \\
\text { diarrea) }\end{array}$ & 60.8 & 34.09 & 68.39 & $* * *$ \\
\hline Cansancio permanente & 42.21 & 22.73 & 47.74 & $* *$ \\
\hline $\begin{array}{l}\text { Consumo excesivo de } \\
\text { alcohol o drogas }\end{array}$ & 1.51 & 0 & 1.94 & ns \\
\hline
\end{tabular}


Tabla 11: Modelo de regresión lineal para la severidad de los padecimientos físicos y psicológicos de la violencia laboral

\begin{tabular}{|c|c|c|c|c|}
\hline \multirow[t]{2}{*}{ Variable } & \multicolumn{2}{|c|}{$\begin{array}{l}\text { Consecuencias } \\
\text { psicológicas }\end{array}$} & \multicolumn{2}{|c|}{ Consecuencias físicas } \\
\hline & Coef. & $\mathrm{P}>\mathrm{t}$ & Coef. & $\mathrm{P}>\mathrm{t}$ \\
\hline Edad & 0.0013 & ns & 0.0047 & 0.007 \\
\hline \multicolumn{5}{|l|}{ Estado civil } \\
\hline Casada/unidas & 0.0698 & 0.059 & -0.5868 & ns \\
\hline $\begin{array}{l}\text { Alguna vez casadas } \\
\text { Tiene hijas/os } \\
\text { No (ref.) }\end{array}$ & 0.0561 & ns & -0.5366 & ns \\
\hline Sí & 0.0089 & ns & 0.0157 & ns \\
\hline Antigüedad en la empresa & 0.0037 & ns & -0.0012 & ns \\
\hline \multicolumn{5}{|c|}{ Número de personas que laboran en la empresa } \\
\hline De 51 a 100 personas & 0.0654 & ns & -0.0485 & 0.302 \\
\hline $\begin{array}{l}\text { Más de } 100 \text { personas } \\
\text { Lugar de trabajo } \\
\text { Oficina (ref.) }\end{array}$ & 0.0026 & 0.941 & -0.0828 & 0.027 \\
\hline $\begin{array}{l}\text { Fábrica } \\
\text { Jornada de trabajo (hrs.) } \\
\text { De } 5 \text { a } 8 \text { hrs (ref.) }\end{array}$ & 0.0231 & ns & 0.0469 & ns \\
\hline De 9 a 12 hrs. & 0.1245 & 0.000 & 0.1255 & 0.000 \\
\hline $\begin{array}{l}\text { Índice de satisfacción con } \\
\text { el espacio laboral }\end{array}$ & 0.0428 & ns & 0.0529 & ns \\
\hline $\begin{array}{l}\text { Índice de satisfacción con } \\
\text { el ambiente laboral }\end{array}$ & 0.0060 & ns & 0.0331 & ns \\
\hline Violencia laboral & 0.1343 & 0.0000 & 0.1309 & 0.000 \\
\hline Constante & 0.0045 & ns & -0.0822 & $\mathrm{~ns}$ \\
\hline $\mathrm{N}$ & & & & 195 \\
\hline $\mathrm{R}^{2}$ & 0.2 & & & 0.2306 \\
\hline $\mathrm{R}^{2}$ ajustado & 0.2 & & & 0.1798 \\
\hline Prob $>F$ & & & & 0.00 \\
\hline
\end{tabular}

Fuente: cálculos propios basados en la encuesta aplicada.

Se observa que la edad de las mujeres de la muestra deja ver que por cada año que ésta se incrementa, el valor en el índice de severidad de padecimientos físicos aumenta en 0.004. Este resultado era esperable, en tanto que las dolencias físicas son bastante más frecuentes entre las personas conforme avanza la edad de estas.

La variable número de personas que laboran en la empresa, muestra que el valor del índice de severidad de los padecimientos físicos disminuye 
en 0.08 para las mujeres que laboran en empresas o instituciones con un número mayor a 100 personas, en comparación con aquellas mujeres que laboran en las empresas e instituciones con hasta 50 personas.

Por otro lado, la jornada de trabajo es también una variable que se relaciona de manera significativa con el índice de padecimientos físicos, por lo que para aquellas mujeres que trabajan una jornada de 9 a 12 horas el valor del índice se incrementa en 0.12 en comparación con aquellas mujeres que laboran de cinco a ocho horas diarias. La explicación de este resultado parece bastante sencilla, ya que jornadas más extensas de trabajo suponen una mayor exigencia física para las trabajadoras, que las harían más vulnerables a malestares físicos.

Por último, se constata que el índice de padecimientos físicos se incrementa en 0.13 , para aquellas mujeres que sufrieron alguna situación de violencia en el ámbito laboral respecto a aquellas mujeres que mencionaron lo contrario (ver Tabla 11).

Se corrobora así que los padecimientos físicos y psicológicos se ven incrementados cuando las mujeres sufren algún tipo de violencia dentro de sus espacios laborales.

\section{Conclusiones}

En México existen pocos datos sobre la violencia que viven las mujeres dentro de los espacios laborales o bien los que existen se encuentran mayormente acotados al acoso, hostigamiento sexual o discriminación por embarazo. Por otra parte, las grandes encuestas sobre violencia contra las mujeres en nuestro país consideran apartados muy pequeños dirigidos a la violencia laboral contra las mujeres, lo que limita el acceso a datos estadísticos sobre esta problemática.

Este trabajo proporciona datos que apuntan a una elevada prevalencia del problema de violencia laboral contra las mujeres mexicanas. En concreto, se observa que la violencia psicológica o emocional es la que presenta una mayor prevalencia, con porcentajes bastantes altos entre las mujeres encuestadas, seguida por manifestaciones de violencia sexual como el acoso de género y la atención sexual no buscada, que presentaron prevalencias elevadas entre las mujeres encuestadas. En específico, el acoso de género muestra mayor prevalencia con 57.79 por ciento de las mujeres que declararon haberlo sufrido alguna vez, seguido de la atención sexual no buscada recibida por 40.7 por ciento de las mujeres de la muestra. Estos dos tipos de violencia son mayormente ejercidos por los compañeros (varones) de las trabajadoras y los datos nos muestran que los comentarios sexistas, 
vulgares, ofensivos, las miradas obscenas e incluso los tocamientos son manifestaciones violentas que tienen una presencia relevante en los centros de trabajo.

Si bien la violencia física y la económica arrojan prevalencias menores en comparación con los otros tipos de violencia, no deja de ser relevante su presencia en los espacios de trabajo, y que probablemente es en realidad algo mayor, considerando que en el cuestionario empleado sólo se incluyó un par de ítems para su identificación.

La información recabada a partir de esta pequeña encuesta permite además identificar algunos factores que incrementan la probabilidad de que las mujeres vivan situaciones de violencia dentro de sus espacios laborales, referidos a la estructura física, el capital de la empresa, y el tipo de lugar de trabajo.

Los resultados derivados de los modelos de regresión logística multivariada aplicados a la violencia psicológica, emocional o mobbing y al acoso de género y a la atención sexual no buscada muestran que el estado civil de las mujeres y el tipo de lugar de trabajo son factores que inciden en el riesgo de que las mujeres sufran alguna situación de violencia de este tipo.

Respecto de los padecimientos físicos y psicológicos que las encuestadas manifestaron haber experimentado derivados de una situación de violencia laboral, el estrés se presenta como uno de los padecimientos más frecuentes, con 82.87 por ciento de las mujeres afirmando haberlo padecido. Si bien este malestar puede derivarse de diversas situaciones o factores no relacionados con la violencia laboral, los datos confirman una prevalencia significativamente mayor en el subconjunto de mujeres que reportaron haber recibido violencia laboral. De la misma manera, todos y cada uno de los padecimientos psicológicos por los que se indagó en la encuesta evidencian que se presentan en un porcentaje significativamente más alto entre aquellas mujeres que han tenido experiencias de violencia laboral, comparadas con mujeres sin violencia laboral en la muestra.

Por otro lado, padecimientos como los trastornos gastrointestinales (colitis, gastritis, entre otros), los dolores de cabeza, migraña, y el cansancio permanente son efectos físicos que las mujeres encuestadas también presentaron con mayor frecuencia derivados de alguna situación de violencia laboral.

Aunque este trabajo presenta resultados limitados, dado que se basan en una muestra pequeña y no representativa de mujeres, deja ver algunas de las características y condiciones que favorecen situaciones de violencia laboral en contra de las mujeres, así como algunos de los efectos físicos 
y psicológicos que pueden presentarse en mujeres que han estado o están en una situación de violencia y cuya severidad se ve incrementada con la presencia de alguna situación violenta.

Creemos que el desarrollo de estudios e investigaciones que recaben nuevos datos de muestras probabilísticas y representativas así como información cualitativa sobre la violencia contra las mujeres en los espacios laborales que den pie a nuevas formas de preguntar sobre esta problemática y sus distintos elementos, favorecerán una comprensión más a fondo de esta problemática, y facilitarán avanzar en la generación de estrategias de prevención y atención de la violencia laboral contra las mujeres.

\section{REFERENCIAS BIBLIOGRÁFICAS}

Alves, J., 2000, "Liderazgo y clima organizacional”, en Revista de psicología del deporte, 9 (12), 123-133.

Archer, David, 1999, "Exploring "bulliying" culture in the para-military organization”, in International Journal of Manpower 20 (1-2), 94-105.

Carvajal Orozco, J. and Londoño, C., 2013, "Violencia en el trabajo: investigaciones realizadas en Colombia”, en Sotavento M.B.A, (22), pp.114-124.

Centro de Estudios para el Adelanto de las Mujeres y la Equidad de Género, 2011, Violencia laboral (mobbing) desde la perspectiva de género. México: Cámara de Diputados, LXI Legislatura, Dirección de Estudios Jurídicos de los Derechos Humanos, Ciudad de México.

Chapell, D. y Di Martino, V., 2006, Violence at work. Génova, Suiza: International Labour Organization.

Díaz, Laura, 2005, "Retrato en sepia: formas de violencia doméstica", en Veredas, vol. 10, Universidad Autónoma Metropolitana, pp.441-460.

Frías, S., 2011, "Hostigamiento, acoso sexual y discriminación laboral por embarazo en México”, en Revista Mexicana de Sociología 73 (2), pp.329-365.

Frías, S., 2013, "Definiciones, género y acoso sexual en el ámbito laboral: el caso de un Tribunal Federal Mexicano", en Agoff, C., Casique, I. y Castro, R. (coord.), Visible en todas partes. Estudios sobre violencia contra mujeres en múltiples ámbitos, Ciudad de México: CRIM-UNAM y Porrúa.

González, G.I., 2018, "La violencia laboral desde una perspectiva de género en la Administración Pública de la provincia de Corrientes”, en Revista Pilquen 21(4), pp.10-21.

Gruber, James E., 1992, “A typology of personal and environmental sexual harassment: research and policy implications for the 1990's”, in Sex Roles 26: 447-464.

Heise, Lori, 1994, Violencia contra la mujer. La cara oculta de la salud, Programa Mujer, Salud y Desarrollo / Organización Panamericana de la Salud (OPM), Washington. 
INEGI, 2011, Encuesta Nacional sobre la Dinámica de las Relaciones en los Hogares 2011. Tabulados básicos: Instituto Nacional de Estadística y Geografía (INEGI).

INMUJERES, 2008, Violencia contra las mujeres: un obstáculo crítico para la igualdad de género. Guía Metodológica para la sensibilización en género: una herramienta didáctica para la capacitación en la Administración Pública, (vol. 4). Instituto Nacional de las Mujeres (INMUJERES), México: Instituto Nacional de las Mujeres.

Lamas, M., 2004, "El problema de ser mujer, visto por mujeres", en Violencia contra la mujer en México. México: Comisión Nacional de Derechos Humanos.

Ley General de Acceso de las Mujeres a una vida libre de violencia. Diario Oficial de la Federación, México, 1 de febrero de 2007.

Milczarek, M., 2010, Workplace Violence and Harassment: a European Picture. Luxemburgo: European Agency for Safety and Health at Work (EU-OSHA).

Ministerio de la Protección Social, 2004, Formas y consecuencias de la violencia en el trabajo. Medellín, Colombia: Ministerio de la Protección Social.

Molina Armenta, A., 2010, Violencia hacia las mujeres en el ámbito laboral en México, El Colegio de México.

OIT, 2017, Informe Mundial sobre la Protección Social 2017-2019: La protección social universal para alcanzar los Objetivos de Desarrollo Sostenible, Oficina Internacional del Trabajo (OIT), Ginebra, 497 p.

Olivares, E. e Inchaústegui, T., 2011, Modelo ecológico: para una vida libre de violencia de género. Comisión Nacional para Prevenir y Erradicar la Violencia contra las Mujeres (CONAVIM). Ciudad de México.

Oliver y Rosavalls, E., 2004, Violencia de género. Investigaciones sobre quiénes, por qué y cómo superarla. Barcelona, El Roure Editorial.

ONU, 2015, Transformar nuestro mundo: la Agenda 2030 para el Desarrollo Sostenible, Naciones Unidas, (ONU), Asamblea General, 18 de septiembre de 2015.

Organización Panamericana de la Salud, 2002, Informe Mundial sobre la violencia y la salud: resumen. Washington, D.C.: Oficina Regional para las Américas de la Organización Mundial de la Salud.

Ortiz, A., 2005, Violencia doméstica: modelo multidimensional y programa de intervención, Universidad Complutense de Madrid.

Pérez, J., y Nogareda, C., 2004, "Violencia en el lugar de trabajo, Centro Nacional de Condiciones de Trabajo. Formas y consecuencias de la violencia en el trabajo", citado en Ministerio de la Protección Social. Formas y consecuencias de la violencia en el trabajo. Medellín, Colombia: Ministerio de la Protección Social.

Zapf, D.; Escartin, J.; Einarsen, S.; Hoel, H. y Vartia, M., 2011, "Empirical findings on bullying in the workplace", in Einarsen, Stale, Hoel, Helge, Zapf, Dieter y Cooper, Cary (eds.). Bullying and harassment in the workplace: Developments in theory, research, and practice. London: Taylor and Francis, pp. 75-105. 
Violencia laboral. Análisis de los factores de riesgo y consecuencias en la vida de las mujeres ... / V.B. CRUZ ARROYO e I. CASIQUE

Zuñiga, Mercedes, 2005, "La invisibilidad de la violencia en la dominación de género en el trabajo”, Debate Feminista, vol. 31, pp. 111-130.

\section{RESUMEN CURRICULAR DE LAS AUTORAS}

Vianney Berenice Cruz Arroyo

Es licenciada en Ciencias Políticas y Administración Pública y estudió la maestría en Estudios Políticos y Sociales. Ambos grados los obtuvo en la Universidad Nacional Autónoma de México. Intelectualmente se interesa en los temas de administración pública, ciencias políticas, género, violencia contra las mujeres, masculinidades, planeación estratégica, evaluación de proyectos, metodología de la investigación. Profesionalmente se desempeña en el Instituto Nacional de Desarrollo Social de la Secretaría de Bienestar, México.

Dirección electrónica: vianncruz04@gmail.com

Registro ORCID: http://orcid.org/0000-0001-5193-8902

\section{Irene Casique}

Estudió la licenciatura en Sociología en la Universidad Católica Andrés Be1lo, Caracas-Venezuela. La maestría en Demografía en El Colegio de México y el doctorado en Sociología/Demografía en The University of Texas at Austin. Actualmente se desempeña como investigadora en el Centro Regional de Investigaciones Multidisciplinarias de la Universidad Nacional Autónoma de México.

Dirección electrónica: irene@correo.crim.unam.mx

Registro ORCID: http://orcid.org/0000-0003-2523-4252

Artículo recibido el 2 de octubre de 2017 y aprobado el 19 de marzo de 2019. 